\title{
$7 \quad$ Auf dem Weg zu einer neuen Theorie
}

\subsection{Eine heuristische Betrachtung als Ausgangspunkt}

\section{Worauf bauen die weiteren Überlegungen auf? ${ }^{130}$}

Von diesem Kap. 7 bis incl. Kap. 9 steht die Suche nach ,Übertragungswegen' der Information im Mittelpunkt und damit die Suche nach Antworten zur vierten Hauptkategorie aus dem selektiven Codierungsprozess. Diese Antworten bilden die Basis auf der eine ,wissenschaftliche Legitimation' aufgebaut sein muss.

Vor der weiteren detaillierten Darstellung des neuen Theoriemodells soll, anhand 12 heuristischer Überlegungen, eine erste Orientierung gegeben werden (Abb. 32). Eine plausible , Theorie zur Funktionsweise von Intuition und SyA' muss Antworten zumindest für elf der zwölf Säulen finden. Allein Säule 12 wäre eine nützliche, aber keine hinreichende Bedingung. Gleichzeitig wird verständlich, wie sich der Gesamtzusammenhang der Argumentationskette darstellen und auf welchen Säulen er aufbauen wird. (Zugehörige Literaturangaben werden in den vertiefenden Kapiteln beigefügt.)

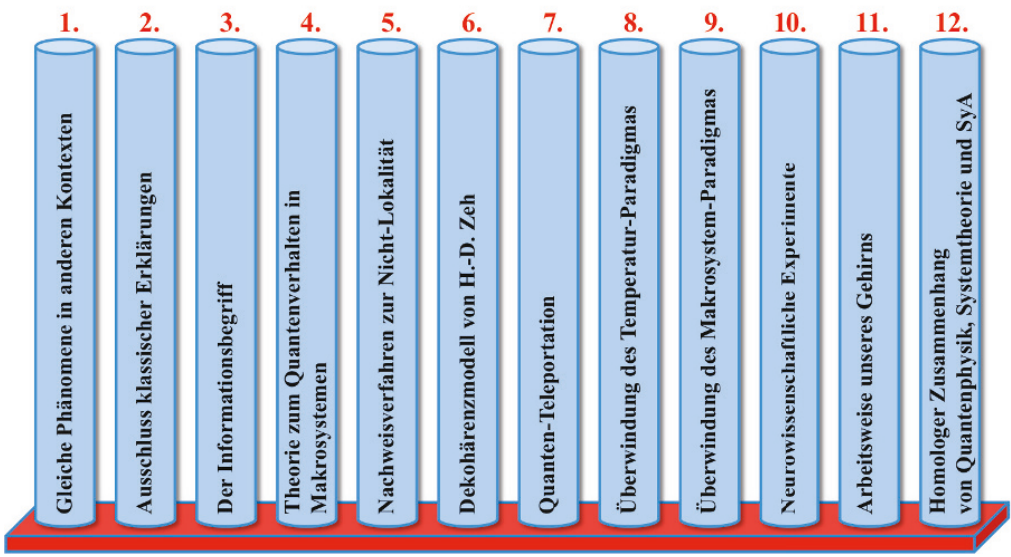

Abb. 32 | Die 12 Säulen zur heuristischen Annäherung an das Gesamtmodell

Diese 12 Säulen bilden die Grundlage dieser Forschungsarbeit, um ein plausibles, belastbares Modell zum Wirk-Prozess von SyA zu entwickeln. Dabei muss jede dieser Säulen bestätigt bzw. falsifiziert werden. (eigene Darstellung)

\footnotetext{
${ }^{130}$ Die folgenden Inhalte sind im Wesentlichen bereits 2015 veröffentlicht worden und werden zum Teil wortwörtlich wiedergegeben (Gehlert 2015a).
}

(C) Der/die Herausgeber bzw. der/die Autor(en) 2020

T. Gehlert, System-Aufstellungen und ihre naturwissenschaftliche

Begründung, Systemaufstellungen in Wissenschaft und Praxis,

https://doi.org/10.1007/978-3-658-29167-9_7 


\section{Säule 1 - Gleiche Phänomene in anderen Kontexten}

Säule 1 zeigt die Ähnlichkeit der Phänomene von SyA in Bezug zur Entscheidungs- und Intuitionsforschung. Ein neues theoretisch fundiertes Modell, welches den zugrundeliegenden Prozess zu beschreiben in der Lage ist, wird auf die gesamte Klasse anwendbar sein. Kurze Beschreibungen und Vergleiche zu anderen, heute noch als merkwürdig angesehenen Phänomenen aus Therapie, Medizin, Beratung, Kommunikation, Mentaltechniken und Bewusstseinsforschung zeigen, dass das Theoriemodell deutlich über die SyA hinaus Gültigkeit entwickeln kann. Dies ist in den vorangegangenen Kap. 3 und 4.1 bereits veranschaulicht worden.

\section{Säule 2-Ausschluss klassischer Erklärungen}

Sie ergibt sich aus dem Ausschluss aller klassischen Erklärungen (wie bereits in Kap. 5 geschehen) und zwingt zur Erweiterung der Perspektive bei der Suche im Möglichkeitsraum.

\section{$\underline{\text { Säule } 3 \text { - Der Informationsbegriff }}$}

Im Übergang von den physikalischen Grundlagen zum Menschen lässt sich Säule 3 positionieren. Hier geht es um den Informationsbegriff wie er mittlerweile in der Physik und in den Geisteswissenschaften verwendet wird. Wie auch hier bereits gezeigt wurde, liegen ausreichende Modelle vor, wie sich eine Anwendung des Informationsbegriffs und der -übertragung auf die Methode der SyA denken lässt. Mit der in Kap. 4.2 vorgenommenen Normierung ist das gleiche Verständnis bzgl. ,Information' auf alle Forschungsgebiete ausdehnbar.

\section{Säule 4 - Theorie zum Quantenverhalten in Makro-Systemen}

Säule 4 wird durch die theoretischen Überlegungen von a) Tegmark und Wheeler, b) Greenstein und Zajonc und schließlich c) von Lucadou gebildet. Die Ersteren bieten eine strukturelle, hierarchische Gliederung, in der das Puzzle verschiedener Wissenschaftstheorien in Beziehung zueinander gesetzt wird. Auf dieser Basis lässt sich die im Weiteren angebotene Theorie der SyA als holistische oder komplementäre Theorie verstehen und bietet keine Möglichkeit eines reduktionistischen Verständnisses. Die Zweiten haben drei Bedingungen formuliert, unter denen quantenphysikalisches Verhalten auch bei makroskopischen Systemen möglich ist. Wie wir noch sehen werden, fallen in diese Kategorie eindeutig die Spezies Mensch sowie alle lebenden Organismen. Von Lucadou schließlich zeigt Rahmenbedingungen auf, unter denen die Experimente (z. B. SyA) durchgeführt werden sollten, bei denen quantenphysikalische Effekte Wirkung entfalten können.

\section{$\underline{\text { Säule } 5 \text { - Nachweisverfahren zur Nicht-Lokalität }}$}

$\mathrm{Zu}$ Beginn der 5. Säule steht eine Frage: Wie kommt es zu Modellbildungen und wie weisen unsere Wissenschaften nach, dass ihre Modelle sinnvolle Beschreibungen der Wirklichkeit darstellen? Betrachten wir die Physik, so orientiert sich diese an beobacht- 
baren Ereignissen, für die sie die dahinter liegenden Prozesse sucht. Als Besonderheit gilt in dieser Disziplin die fast ausschließlich mathematische Behandlung der Annahmen. Ohne eine solch mathematisch plausible Beweisführung finden die Modelle keine Akzeptanz - so nimmt man zumindest üblicherweise an.

Tatsächlich braucht es auch in der Physik noch die Experimente, die die mathematische Beweisführung stützen oder widerlegen. Auf diese Weise gelang es schließlich, Fragestellungen, für die keine Erklärungen im Rahmen der klassischen Physik zu finden waren, zu überwinden und die Quantenphysik zu entwickeln. Dass die Kombination von Experimenten und Mathematik auch in der Physik nicht immer die letzte Frage zu beantworten vermag, wird in der gegenwärtigen Situation deutlich. Denn die heutigen quantenphysikalischen Theorien bieten zumindest zwei grundverschiedene mathematische Ansätze, die beide zu den gleichen präzisen Ergebnissen für die damit verbundenen Experimente führen, allerdings mit absolut gegenläufigen Theorieansätzen. Zum einen das 1932 von John von Neumann formulierte Modell auf das sich die Kopenhagener Interpretation bezieht (Neumann 1996: 101-157) und zum anderen die De-BroglieBohm-Theorie oder auch Bohmsche Mechanik genannt (Goldstein 2017; Passon 2010). Experimentell lassen sich beide Ansätze nicht unterscheiden.

Darüber hinaus gibt es eine Vielzahl von Ansätzen, wobei jede für sich noch Fragen unbeantwortet lässt (z. B. Stringtheorie, M-Theorie, Vielwelten-Theorie). Einerseits hatte dies nun zur Konsequenz, dass weiterhin intensiv nach Modellen gesucht wird, die die scheinbaren Widersprüche auflösen. Andererseits wird in den Argumentationen und Positionierungen so getan, als ob schon alles klar und bewiesen wäre. So vermitteln Blog-Beiträge oder manche Bücher den Eindruck, als ob die jeweils andere Seite völlig ahnungslos oder unwissend wäre (Hümmler 2017). Dies ist mitnichten so, wie die Entwicklungen in Quanten-Biologie und -Chemie aktuell zeigen und konsequenterweise existiert damit auch ein unbekannter Raum für Neues. Dazu passen auch die Ansichten von Heisenberg (Hägele 2000: 22) und Zeilinger (2002), nach der die Naturwissenschaften uns nur ein Bild unserer Kenntnis von der Natur vermitteln und nicht ein Bild der Natur selbst.

Das Nachweisverfahren einer Verschränkung und der damit verbundenen Nicht-Lokalität, eines der zentralen Phänomene der Quantenphysik, stellt deshalb die 5. Säule dar. Mit diesem Punkt wird ein weiteres Grundproblem deutlich: Dass sich viele mathematisch behandelbare Phänomene einer direkten Beobachtung entziehen. So kann die Physik heute noch nicht erklären, wie der Raum bzw. die Verbindung innerhalb eines verschränkten Systems zu beschreiben ist. Allerdings kann beobachtet werden, dass die beiden verschränkten Systeme etwas Gemeinsames aufspannen. Die Unterscheidung, ob Systeme ${ }^{131}$ verschränkt sind oder nicht, lässt sich also nicht direkt beobachten, sondern nur indirekt über ihr Verhalten bestimmen. Dazu wird üblicherweise ein mathematisches Verfahren, die Wahrscheinlichkeitsberechnung, herangezogen.

${ }^{131}$ Gewöhnlich bezieht sich der Physiker dabei auf Teilchen und physikalische Objekte. 
Analog dazu dienen auch in den Wirtschaftswissenschaften, der Soziologie, Psychologie und Medizin Wahrscheinlichkeitsbetrachtungen zur Bestimmung von Zusammenhängen und zur Verifikation von Modellen bzw. Behandlungsmethoden. Nun existiert auch für SyA eine Wahrscheinlichkeitsuntersuchung, die sozial- und naturwissenschaftlichen Ansprüchen genügt (Schlötter und Simon 2005). Es konnte dabei die Wiederholbarkeit von Wahrnehmungen im Rahmen von SyA eindeutig nachgewiesen werden und diese Ergebnisse lassen keinen Spielraum für einen Zufall.

\section{Säule 6 - Dekohärenzmodell von H.-D. Zeh}

Säule 6 basiert auf dem Dekohärenzmodell von Zeh, das von einer allgemeinen Verschränkung aller Entitäten ausgeht. Die in diesem Modell vorhandene Beobachtungsproblematik bei Makrosystemen wird sich als Sonderfall herausstellen, der behandelbar ist.

\section{Säule 7 -Quanten-Teleportation}

Die 7. Säule wird durch den Versuchsaufbau von Zeilinger zur Quanten-Teleportation repräsentiert, bei dem die eben erwähnte ,Verschränkung' eine zentrale Rolle spielt. Eine analoge Struktur finden wir bei SyA als auch bei zahlreichen anderen Phänomenen. Sie gehören alle der gleichen Klasse an, nämlich einer spezifischen Form von Informationswahrnehmung, für die es heute noch keine grundlegende Erklärung gibt.

\section{Säule 8 - Überwindung des Temperatur-Paradigmas}

Der oben angeführte Versuchsaufbau zur Quanten-Teleportation liefert die Vorlage für die 8. Säule, nämlich die Überwindung des Paradigmas der Notwendigkeit sehr niedrigen Temperaturen, um Quantenverschränkungen messbar und damit beobachtbar zu machen. Die meisten quantenphysikalischen Versuche fanden bei Tiefsttemperaturen statt, welche zur Isolierung der untersuchten Quantensysteme von ihrem Umfeld nötig sind. Man ging in der Dekohärenztheorie davon aus, dass die Umweltbedingungen einen sofortigen Verlust beobachtbarer Quanteneigenschaften aufgrund von Wechselwirkungen nach sich ziehen würden. Dass dem nicht so ist, konnten neueste Experimente eindrucksvoll bestätigen.

\section{$\underline{\text { Säule } 9 \text { - Überwindung des Makrosystem-Paradigmas }}$}

Womit wir zur 9. Säule kommen: Der Überwindung des Paradigmas, dass Makrosystemen, wie beispielsweise Zellen, wegen der angenommenen Wechselwirkungen mit der Umwelt, keine Quanteneigenschaften zeigen. Durch die Arbeiten von Physikern und Biologen konnten solche Eigenschaften mittlerweile nicht nur in Bakterien nachgewiesen werden. Einher geht ein Shift im Verständnis von geschlossenen hin zu offenen Quantensystemen. 


\section{Säule 10 - Neurowissenschaftliche Experimente}

Und schließlich Säule 10: Sie basiert auf der Basis neuer neurowissenschaftlicher Experimente, die EEG-Korrelationen zwischen Menschen incl. des Einsatzes eines Faraday'schen Käfigs zeigen konnten. Mithilfe des Faraday'schen Käfigs bzw. abgeschirmter Räume scheiden in bestimmten Situationen normale EM-Wellen als Erklärung aus. Unterstützung für Säule 10 liefern zudem die Erkenntnisse aus der SpiegelneuronenForschung.

\section{$\underline{\text { Säule } 11 \text { - Arbeitsweise unseres Gehirns }}$}

Die 11. Säule stellt alternative Modelle zur Arbeitsweise des Gehirns vor, nach denen auch quantenphysikalische Aktivitäten in unserem Gehirn angenommen werden können. Darüber hinaus lassen Entwicklungen im Bereich von Quantencomputern die alternativen Modelle sehr plausibel erscheinen; dort speziell die Möglichkeit von Fehlerkorrekturen und das Erzeugen stabiler Kohärenz. Hier können die biologische Struktur des Gehirns und theoretische und experimentelle Ergebnisse der Quantenphysik in einer Form in Beziehung gebracht werden, bei der auch für das Gehirn quantenphysikalische Prozesse als wahrscheinlich erscheinen.

\section{Säule 12 - Homologer Zusammenhang von Quantenphysik, Systemtheorie und SyA}

Last but not least führt der Vergleich von Quantenphysik, Systemtheorie und SyA zu Säule 12. Eine unglaubliche Ähnlichkeit, um nicht zu sagen Synchronizität der Grundprinzipien der verschiedenen Disziplinen unterstützen die Annahme, dass wir es hier nicht nur mit Analogien, sondern mit einem fundamentalen Zusammenhang zu tun haben.

Säule 1 bildete den Start, indem sie Parallelitäten in Bekanntem sichtbar werden ließ. Sie macht deutlich, dass SyA mitnichten so einzigartig sind, wie sie heute noch erscheinen mögen. Säule 2 und 3 erforschten vorhandene Erklärungsoptionen und arbeiten Schwachstellen und Möglichkeitsräume heraus. Die Säulen 4-8 folgen im Prinzip einer Logik vom Mikro- zum Makro-Kosmos. Soll heißen: Die Säulen legen die weitere Grundlage, wobei ausschließlich die Quantenphysik im Fokus steht. Die Säulen $9-11$ bilden den Übergang zu lebenden Systemen. Basieren die ersten 10 Säulen dieser heuristischen Zusammenfassung im Wesentlichen auf den Ergebnissen und Überlegungen der Forscher aus den verschiedenen Wissenschaften, so wird bei der 10. und 11. Säule zwar auf vorhandener Forschung aufgesetzt, ergänzend aber eigene weiterführende Ideen und Interpretationen entwickelt. Säule 12 schließt mit der Untersuchung der Beziehungen der zentralen Theoriekonzepte ab, die dieser Forschung zugrunde liegen.

Des Weiteren werden auch hier am Ende jedes Kapitels sofort die Bezüge herausgearbeitet, die mit speziellen Aspekten der SyA in Zusammenhang zu bringen sind. Gegen Ende der Arbeit werden diese der besseren Übersicht wegen noch einmal in Kap. 9 zusammengefasst. 


\subsection{Ausgangsbasis für eine komplementäre Theorie der SyA}

SyA lassen sich nur unter Berücksichtigung verschiedener Forschungsgebiete adäquat beschreiben

Ausgehend von den bisherigen Ergebnissen und den aus verschiedensten Forschungszweigen zusammengetragenen experimentellen und theoretischen Erkenntnissen wird im Folgenden ein Gesamtzusammenhang entworfen, der anschließend detailliert aufbereitet und untermauert wird.

An dieser Stelle sei noch eine persönliche Anmerkung erlaubt:

Eine interessante Erfahrung bei meiner Forschung zu Erklärungen der Aufstellungsarbeit waren die unterschiedlichen Reaktionen meiner Gesprächspartner. Je nachdem aus welcher Wissenschaftsdisziplin sie kamen, waren die Erklärungsversuche, fast möchte man sagen natürlicherweise, völlig unterschiedlich. Von einem völligen Verzicht auf die Notwendigkeit einer fundierten Erklärung bis hin zu Ansätzen aus der eigenen Profession. Der ,verrückte' Ansatz, SyA aus der Physik besser aus der Quantenphysik heraus erklären zu wollen, stieß nur bei sehr wenigen auf Verständnis, inklusiv den Physikern selbst.

Neben dem grundsätzlichen Problem, dass Menschen und lebende Systeme im allgemeinen Verständnis der Physik als makroskopische Systeme betrachtet werden, in denen sich keine Quanteneffekte zeigen können, war der Hinweis, dass eine quantenphysikalische Erklärung ein eher reduktionistischer Ansatz wäre, eine weitere gewichtige Rückmeldung.

In der Tat liefert die Quantenphysik nicht alles, was für ein vollständiges Verständnis von SyA im Auge zu behalten ist - allerdings kann sie wohl doch mehr erklären, als ihr üblicherweise zugeschrieben wird. Im Folgenden soll deshalb ein Bogen, ausgehend von der Quantenphysik, über verschiedene Wissenschaften gespannt werden, aus denen Antworten abgeleitet werden, um das Phänomen von SyA in seiner Gänze zu verstehen und zu interpretieren. Verschiedene Formen der Darstellung sollen helfen die Gleichstellung und Zusammenhänge dieser Wissenschaftstheorien anschaulich zu machen. Zur Vermeidung von Redundanzen wird bis auf Weiteres überwiegend nur noch auf SyA Bezug genommen. Sie soll stellvertretend für den weiten Bereich der Intuitionsphänomene stehen.

Beginnen wollen wir bei der Physik. Hier ist es in keiner Weise so, dass führende Köpfe sich nur auf Rechenoperationen fokussieren und keinerlei Versuch unternehmen würden, ihre Erkenntnisse in einen Gesamtzusammenhang bringen zu wollen. So haben Tegmark und Wheeler (Tegmark und Wheeler 2001) eine strukturelle, hierarchische Gliederung vorgestellt, in der das Puzzle verschiedener Wissenschaftstheorien in Beziehung zueinander gesetzt wird (Abb. 33). 


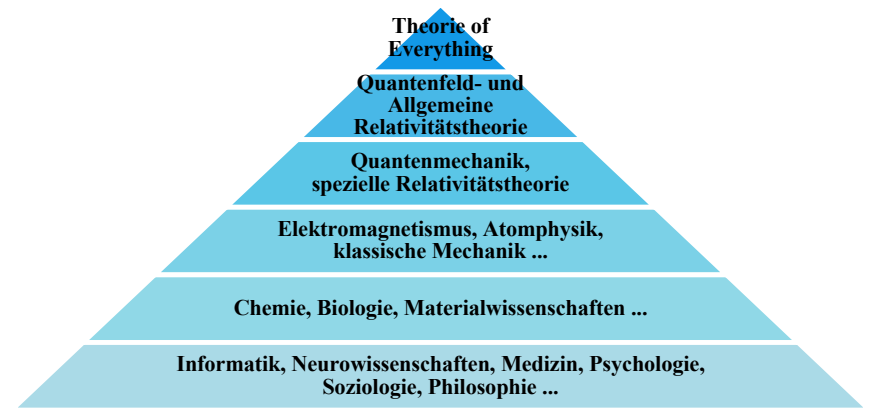

Abb. 33 | Hierarchische Gliederung der Wissenschaftstheorien die sich von oben nach unten immer weiter aufteilen und letztlich immer neue, emergente Phänomene behandeln. In Anlehnung an Tegmark und Wheeler (Tegmark 2008: 103; Tegmark und Wheeler 2001).

Nach ihnen lassen sich Theorien ,,grob in einem hierarchischen Schema anordnen, wobei sich jede - zumindest im Prinzip - von fundamentaleren Theorien herleitet, die in der Hierarchie über ihr stehen. [...] All diese Theorien enthalten zwei Komponenten: mathematische Gleichungen sowie Worte, die erklären, wie die Gleichungen mit experimentellen Beobachtungen zusammenhängen. [...] Auf jeder Stufe der Theorie-Hierarchie werden neue Begriffe - wie Proton, Atom, Zelle, Organismus, Kultur - eingeführt, weil sie auf bequeme Weise das Wesentliche einfangen, ohne auf die übergeordneten Theorien zurückgreifen zu müssen. Im Großen und Ganzen nimmt das Verhältnis von Gleichungen zu Worten ab, wenn man in der Hierarchie abwärts schreitet, und fällt für sehr anwendungsnahe Gebiete wie Medizin und Soziologie fast auf null. Hingegen sind die Theorien in der Nähe des Gipfels fast reine Mathematik, und die Physiker kämpfen noch immer darum, die mathematisch verschlüsselten Begriffe zu verstehen “ (Tegmark und Wheeler 2001).

Diese auf den ersten Blick reduktionistische Darstellung wandelt sich bei genauer Betrachtung in eine holistische ${ }^{132}$, wie im Folgenden deutlich wird oder in eine komplementäre, wie sie aus der Quantenphysik bekannt ist und dort von Niels Bohr eingeführt wurde. ,, dass gerade das Grundpostulat der Unteilbarkeit des Wirkungsquantums vom klassischen Standpunkt aus ein irrationales Element darstellt, das unvermeidlich einen Verzicht fordert hinsichtlich der Kausalitätsbeschreibung in Raum und Zeit und infolge der Zusammenkettung zwischen Erscheinung und Beobachtung uns auf eine Beschreibungsweise hinweist, die in dem Sinne als komplementär bezeichnet wird, dass jede gegebene Anwendung von klassischen Begriffen den gleichzeitigen Gebrauch von anderen klassischen Begriffen ausschließt, die in anderem Zusammenhänge gleich notwendig für

${ }^{132}$ Holistisch meint in diesem Zusammenhang eine ganzheitliche Erklärung, bei dem Erscheinungsformen und Erklärungen aus unterschiedlichen Kontexten in Verbindung miteinander gebracht werden. Erscheinungsformen, die je Kontext individuell beschrieben werden müssen, aber in Summe eine Ganzheit hervorbringen ohne als Summe dieser Teile verstanden werden zu können. 
die Beleuchtung der Erscheinungen sind“ (Bohr 1931: 6). „In dieser Verbindung muss jedoch nicht vergessen werden, dass es bei der Zusammenfassung der physischen und psychischen Seite des Daseins sich um ein besonderes Komplementaritätsverhältnis handelt, das sich nicht erschöpfend mittels einseitig physikalischer oder psychologischer Gesetzmäßigkeiten veranschaulichen lässt “(Bohr 1931: 15). Aus diesen Ausführungen geht hervor, dass Bohr den Begriff ,Komplementarität' über den Zusammenhang Welle-Teilchen ${ }^{133}$ hinaus verwendete. Komplementär bedeutet in diesem Sinne, dass sich bei der Beobachtung eines Geschehens, unterschiedliche Blickwinkel gegenseitig ausschließen, dennoch zusammengehören und einander ergänzen. Das bedeutet, dass wir je Untersuchungssituation und verwendeter Methoden nur einen der komplementären Begriffe anwenden können und jeweils individuelle Ergebnisse aus dieser Untersuchung erhalten. In unserem Fall gehen mit den unterschiedlichen Blickwinkeln unterschiedliche Methoden einher, so wie es für das zweite Zitat von Bohr auch notwendig wird. Der Zusammenhang kann demzufolge auch nur erkannt werden, wenn die verschiedenen Perspektiven in Beziehung zueinander gesetzt werden, was zwingend eine disziplinübergreifende Forschung und Theoriebildung nach sich zieht. Fahrenberg ${ }^{134}$ bezeichnet ,Komplementarität' als Meta-Relation „um verschiedene Bezugssysteme zu kombinieren oder zu vereinigen " (Fahrenberg 2013: 288). Aus sich heraus bleiben die Einzelergebnisse der jeweiligen Forschungsdisziplinen Stückwerk, gänzlich unverständlich oder täuschen eine Scheinplausibilität vor, die aus einer übergeordneten Perspektive nicht hält, was sie verspricht. Zur Wirkung kommt eine Variante von Kahneman's ,theorieinduzierte Blindheit' (Kahneman 2016), sofern man sich nur auf einer theoretischen Betrachtungsebene bewegt.

Insofern bietet sich eine alternative Darstellung an, die den hierarchischen Eindruck von Abb. 33 auflöst und einen Zusammenhang veranschaulicht, der die Wissenschaften in einen emergenten Zusammenhang bringt (Abb. 34). Ausgehend von einem naturwissenschaftlich verankerten Zentrum emergieren neue Systeme mit immer spezifischeren Phänomenen, die in jeweils anderen Wissenschaften behandelt werden müssen. Physikalisch kann man hier von einer Symmetriebrechung (Penrose 2010: 324) sprechen, bei der sich übergeordnete Symmetrien (geschlossene Zusammenhänge) in immer neue Teilsymmetrien (wieder in sich geschlossene Zusammenhänge) ausdifferenzieren. Das für die Emergenz typische Erscheinungsbild, dass das ,Ganze' Eigenschaften besitzt, die nicht in den Teilen zu beobachten sind (Drossel 2016: 8), zwingt zu neuen Modellen, Untersuchungsmethoden und Vorgehensweisen. In den jeweiligen Verzweigungen las-

${ }^{133}$ Jordan (Jordan 1938: 110) hat den Komplementaritätsbegriff in Zusammenhang mit dem Welle-Teilchen-Dualismus sehr schön herausgearbeitet: „Die mit der Wellennatur des Lichtes verknüpften Eigenschaften einerseits und die mit der korpuskularen Natur des Lichtes andererseits verknüpften Eigenschaften sind, komplementär ' zueinander in dem Sinne, dass sie niemals in einem und demselben Experiment zugleich in Erscheinung treten (und dann in einen wirklichen unmittelbaren Widerspruch geraten) können “ (in: Fahrenberg 2013: 324).

${ }^{134}$ Das Thema ,Komplementarität' wird ausführlich bei Fahrenberg behandelt (Fahrenberg 2013). 
sen sich Konzepte und Gesetze beschreiben, die jeweils nur für diese Gültigkeit besitzen.

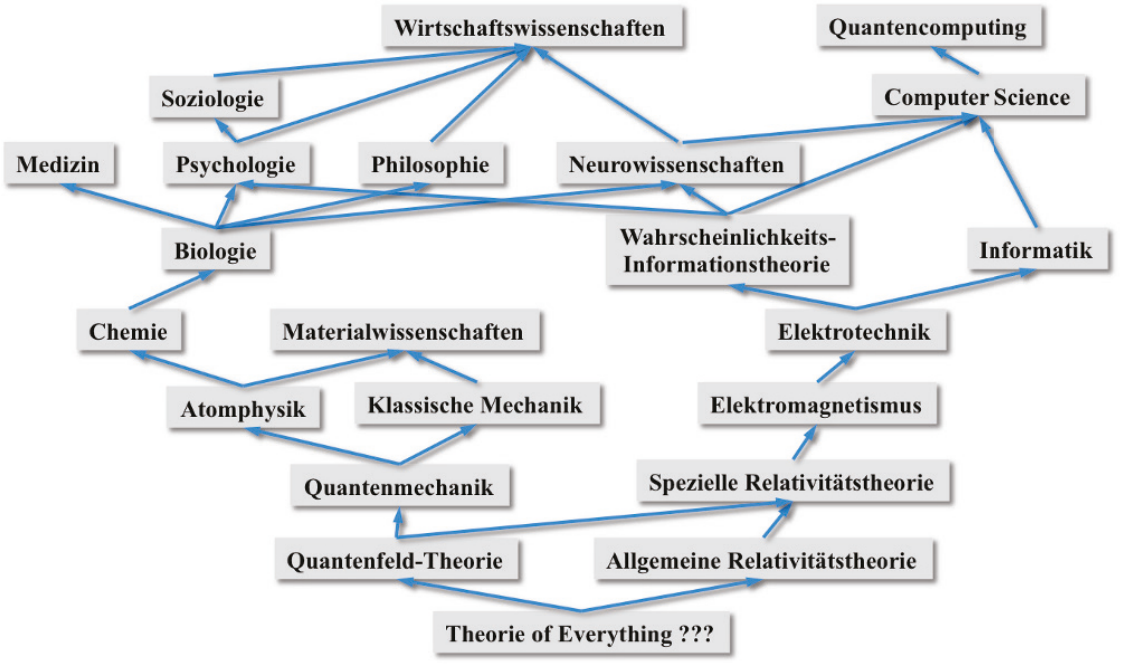

Abb. 34 | Verbindungen zwischen den Wissenschaftstheorien mit Übergangssprüngen

Veranschaulicht werden die Emergenz der Phänomene und eine Auswahl an zugehörigen Wissenschaften, die in einem komplementären Zusammenhang zueinander stehen. Im Gegensatz zu dem scheinbar kontinuierlich sich entwickelnden klassischen Stammbaum ergeben sich bei genauer Betrachtung Sprünge bei den Übergängen, bei denen Phänomene erscheinen, die in der jeweils vorgeschalteten Ebene noch nicht enthalten waren und durch diese nicht vollständig erklärt werden können. Eigene Darstellung in Anlehnung an Tegmark (Tegmark 2008: 103).

Ein Gesamtverständnis ergibt sich deshalb nicht, wenn ich vom Einzelnen auf das Ganze schließe (Induktion) oder vice versa (Deduktion), wohl aber, wenn die verschiedenen Phänomene, Konzepte und Modelle miteinander in Verbindung gebracht werden. Im Prinzip sollte man dabei erwarten, dass die Gesetzmäßigkeiten eines Ausgangspunktes auch auf die entfernteren Phänomene und Wissenschaften zutreffen und Wirkung entfalten (Drossel 2016: 10). Mit jeder Schicht bildet sich zudem eine immer komplexer werdende Realität aus, die sich rein linearen, deterministischen Entwicklungen entzieht, wie es bereits in Kap. 3.1 herausgearbeitet wurde. In diesem Sinn definieren physikalische Gesetze nur ,, den äußeren Bereich von möglichen Prozessen in Natur und Gesellschaft " (Ebeling und Scharnhorst 2015: 427) ohne die SyA und Intuition nicht verstanden werden kann. Gleichzeitig können physikalische Gesetze soziale Prozesse nicht vollständig abbilden oder soziale Prozesse physikalische Gesetze verletzen (vgl. ebd.). „Physikalische, chemische, biologische und soziale Gesetzmäßigkeiten bilden ineinander geschachtelte Trichter, die jeweils die Randbedingungen festlegen, unter denen die immer komplexer werdenden Systeme operieren " (Maturana \& Varela 1987 in ebd.). 
Ausgehend von diesem komplementär-theoretischen Verständnis können fünf Wissenschaftsdisziplinen identifiziert werden, die wesentliche Beiträge zur Erklärung von SyA liefern. Es sind dies Physik, Biologie, Neurowissenschaften, Psychologie und Soziologie (Tab. 13). Würde nur auf eine dieser fünf Hauptkategorien verzichtet werden, fiele die logische Kette einer in sich geschlossenen Theorie der SyA in sich zusammen, wäre nicht nachvollziehbar und damit angreifbar.

Tab. 13 | Wissenschaften mit Beiträgen zur Erklärung von SyA

Diese fünf Wissenschaften sind notwendig, um eine Theorie der SyA beschreiben zu können. (eigene Darstellung)

\begin{tabular}{|c|c|c|}
\hline Wissenschaft & Beitrag zur Erklärung & $\begin{array}{l}\text { Betroffene } \\
\text { Unterkategorie }\end{array}$ \\
\hline Physik & $\begin{array}{l}\text { Erklärt die Basismechanismen, } \\
\text { sozusagen die Grundprinzipien } \\
\text { und damit den Ausgangspunkt. } \\
\text { Die auftretenden Phänomene } \\
\text { lassen sich vollständig nur auf } \\
\text { dieser Ebene beschreiben. }\end{array}$ & $\begin{array}{l}\text { Quantenfeldtheorie und Quan- } \\
\text { tenmechanik } \\
\text { Kohärenz, Verschränkung, Su- } \\
\text { perposition und Nicht-Lokalität }\end{array}$ \\
\hline Biologie & $\begin{array}{l}\text { Erklärt wie Quantenprozesse in } \\
\text { biologischen Systemen wirken } \\
\text { bzw. sich koppeln. }\end{array}$ & $\begin{array}{l}\text { Quantenbiologie } \\
\text { (Biochemie, entsprechend dem } \\
\text { klassischen Verständnis, ist ver- } \\
\text { mutlich nicht ausreichend) }\end{array}$ \\
\hline $\begin{array}{l}\text { Neurowissen- } \\
\text { schaften }\end{array}$ & $\begin{array}{l}\text { Erklärt wie elektromagnetische } \\
\text { bzw. elektrochemische Impulse } \\
\text { und Informationen in Menschen } \\
\text { Prozesse in Gang setzen. }\end{array}$ & $\begin{array}{l}\text { Arbeitsweise des Gehirns, Ge- } \\
\text { hirnwellen und Spiegelneuronen }\end{array}$ \\
\hline Psychologie & $\begin{array}{l}\text { Beschäftigt sich mit den persön- } \\
\text { lichen Voraussetzungen und } \\
\text { Einfärbungen. }\end{array}$ & $\begin{array}{l}\text { Wahrnehmungs- und Aufmerk- } \\
\text { samkeitsforschung } \\
\text { Framing, Priming, Verzerrungen }\end{array}$ \\
\hline Soziologie & $\begin{array}{l}\text { Erklärt wie soziale Systeme mit } \\
\text { abstrakter Information umgehen } \\
\text { und wie es zu den dann folgen- } \\
\text { den Interpretationen kommt. }\end{array}$ & $\begin{array}{l}\text { Hermeneutik, } \\
\text { Konstruktivismus und } \\
\text { Kybernetik } \\
\text { (Letztere ließe sich allerdings } \\
\text { auch bei den anderen Wissen- } \\
\text { schaftstheorien zuordnen) } \\
\text { generalisierte Q-Theorie }\end{array}$ \\
\hline
\end{tabular}

Mit diesen Überlegungen wird auch eine Antwort auf die in den Wissenschaften so gerne geforderte Berücksichtigung von Ockhams Rasiermesser (Rothman 2011) geliefert. 


\section{„Entia non sunt multiplicanda praeter neccessitatem. “ \\ „Entitäten dürfen nicht über das Notwendige hinaus vermehrt werden. “ \\ William van Ockham (1285-1347) \\ (in Clauberg 1654: 320)}

Sie wird als eine der Prämissen guter wissenschaftlicher Arbeit angesehen. Dieses heuristische Forschungsprinzip der Scholastik fordert Sparsamkeit bei der Verwendung von Theorien und damit das Wegschneiden überflüssiger Anhängsel. Also die Reduzierung auf die passendste und vor allem einfachste Erklärung, die möglich ist. Als Einschränkung dieser Forderung lässt sich ausführen, dass sie nur Sinn macht, wenn zwischen mehreren Theorien, die den Sachverhalt zu beschreiben vermögen, gewählt werden kann. Genau diese Bedingung liegt im Fall von Intuition und SyA nicht vor. Zwar lassen sich bei der Intuition einige Phänomene in scheinbar einfacher Weise erklären, dies gilt mitnichten für alle.

Es existiert derzeit keine einfache Erklärung im Untersuchungsfeld der SyA, die sich auf ein Wissenschaftsgebiet reduzieren ließe. Dies sei nochmals veranschaulicht durch die Art der bisherigen Annäherung an das Phänomen SyA. Die Mehrheit der bisherigen Erklärungsversuche kommt aus dem Feld der Soziologie: ,Raumsprache', ,Topologischer Ansatz', ,repräsentierende Wahrnehmung' versuchen auf unterschiedlichen Wegen der Phänomene habhaft zu werden. Aus der Biologie werden ,morphogenetische Felder' und daran angelehnt, wissende Felder' angeboten. Schließlich liefert die Physik erste Ansätze in Verbindung mit dem ,Psi- oder Vakuum-Feld'. Wie bereits ausgeführt, bestehen in allen Beschreibungen erhebliche Schwächen. Entweder sind sie nicht-ausreichend, nicht-zutreffend, nicht-akzeptiert oder sie sind nur Etikette ohne Inhalt, mithin fehlt die geforderte ,wissenschaftliche Legitimation' aus Hauptkategorie 1 und Hypothese 1 .

Wollen wir uns den Prozess bildlich vorstellen, so bietet sich als geeignete Metapher die Zwiebel an. Schale für Schale muss abgetragen werden, um zum Kern vorzustoßen. Dennoch ist die Zwiebel ohne ihre Schalen nicht vollständig. Folgen wir den psychologischen und soziologischen Erklärungen, stellen wir fest, dass wesentliche Phänomene nicht erklärt werden können. Das Gleiche passiert mit Beschreibungen der Neurowissenschaften. Auch hier bleiben Fragen unbeantwortet. Und so stoßen wir Schicht für Schicht auf immer tiefere verborgene Fragen und Mechanismen, bis wir schließlich bei der Quantenphysik enden. Tatsächlich scheint es heute so, dass wir auch quantenphysikalische Konzepte zur vollständigen Beschreibung des Informationstransfers bei SyA heranziehen müssen.

Das was Menschen letztlich als Phänomene und Ergebnisse von SyA wahrnehmen, entfaltet sich vom Zentrum her, Schale für Schale nach außen gerichtet, bis sie sich schließlich in der Makrowelt manifestieren. Die Quantenphysik stellt den Ausgangspunkt für die wahrnehmbaren Phänomene dar und damit für alles was danach kommt. Ohne sie gibt es keinerlei Möglichkeit die Phänomene vollständig zu beschreiben, denn die aktuellen Darstellungen liefern, wenn man sie genau betrachtet, wie wir es getan 
haben, keine tragfähigen Modelle. Die vier weiteren Wissenschaftsrichtungen sind zwingend nötig, um zu verstehen, wie sich Information in uns zu wahrnehmbaren Wirkungen und letztlich zu interpretiertem Sinn entwickelt. Aufgrund dieser Zusammenhänge wird auch verständlich, weshalb sich ein narrativer Ansatz besser zur Beschreibung des untersuchten Gegenstandes (Wirkungsweise der SyA) eignet, als es ein mathematischer Ansatz könnte. Zum einen existieren keine mathematisch-übergreifenden Modelle zur Beschreibung und zum anderen bestünde die gleiche Problematik wie sie die GQT erlebt. In diesem Ansatz werden physikalische-mathematische Formalismen auf soziologische Fragen angewandt, ohne sich auf die Physik selbst beziehen zu wollen. Als Folge erscheint dieser Zugang den meisten derzeit noch wenig plausibel.

Aus diesen Überlegungen ergibt sich eine klare Antwort auf die Frage, ob eine quantenphysikalische Erklärung ein eher reduktionistischer Ansatz wäre. NEIN, selbst unter ausschließlicher Berücksichtigung der Quantenphysik läge keine reduktionistische Beschreibung vor, denn die Quantenphysik selbst ist von Haus aus schon keine Wissenschaft, die reduktionistische Ergebnisse liefert. Und NEIN, weil die anderen Wissenschaften zwingend mit eingebunden werden müssen, will man eine adäquate Beschreibung des Geschehens liefern. Die Symmetriebrüche und die immer wieder emergenten Erscheinungen innerhalb der neuen Teilsymmetrien erfordern eine eigenständige Berücksichtigung. Jede dieser Perspektiven muss ihren Beitrag leisten, um das Gesamte verstehen zu können. Das Gesamtverständnis kann sich dann einstellen, wenn die Fachgebiete mit einem UND verbunden statt mit einem ENTWEDER-ODER getrennt werden.

Diese Überlegungen möchte ich mit einem Zitat von Feynman abschließen, das unsere Ausgangsposition sehr treffend beschreibt, wie ich finde:

„,Wir Physiker haben uns mit diesem Problem [Warum die Natur sich so verhält wie sie sich verhält? Anmerkung des Autors] herumschlagen und einsehen müssen, dass es nicht darauf ankommt, ob uns eine Theorie passt oder nicht, sondern darauf, ob die Theorie Vorhersagen erlaubt, die mit dem Experiment übereinstimmen " (Feynman 1992: 21).

\subsection{Bedingungen für Quantenverhalten in Makrosystemen}

\section{Wie es doch gehen könnte!}

Wie bereits erwähnt, betrachten die meisten Physiker lebende Systeme wie Zellen, Pflanzen, Tiere oder Menschen als Makrosysteme, bei denen sich keine quantenphysikalischen Überlagerungszustände beobachten lassen. So liegt für Zeilinger das Problem nicht in der Übertragung von Quanteneigenschaften auf den Makrokosmos bzw. dass solche Eigenschaften im Makrokosmos nicht existieren würden, sondern in der Schwierigkeit, diese mit unseren beschränkten Möglichkeiten wahrzunehmen (Zeilinger 2005: 102). Unter dieser Voraussetzung entzögen sich Verschränkung und Nicht-Lokalität unserem alltäglichen Zugriff. 


\subsubsection{Drei Bedingungen nach Greenstein und Zajonc}

Lösen wir uns zunächst aber vom Anspruch der Wahrnehmung und betrachten nur die Möglichkeit quantenphysikalischer Mechanismen in makroskopischen Systemen so stoßen wir auf die Arbeiten von Greenstein und Zajonc. Ihre Überlegungen lassen sich auch als Vorlage für einen ersten Plausibilitätscheck verwenden.

Greenstein und Zajonc beschreiben drei Bedingungen, die zu erfüllen sind, damit auch in makroskopischen Systemen quantenphysikalisches Verhalten möglich (Greenstein und Zajonc 2005: 203-205) ist.

1. „The macroscopic variable must be well decoupled from the motion of the microscopic degrees of freedom.

2. The motion of the macroscopic variable must be controlled by a microscopic energy.

3. The temperature must be low. “

Wie wir gleich sehen werden, lassen sich die Punkte 1 und 2 relativ leicht auf lebende Systeme wie Menschen, ja sogar bis hin zu Organisationen übertragen.

\section{Untersuchen wir zunächst Bedingung 1:}

Greenstein und Zajonc führten als Beispiel das Zusammenspiel von Atomen und schwingendem Pendel an. Die Atome befinden sich in ihrer eigenen mikroskopischen Bewegung. Das von ihnen aufgebaute und schwingende Pendel ist mit seiner Gesamtmasse jedoch primär nicht von dieser Mikrobewegung, sondern von der Gravitation abhängig. Das Pendel ist somit von der inneren Bewegung entkoppelt. Dieser Zusammenhang lässt sich auch auf Menschen übertragen.

Der Mensch als Ganzes (Körper und Geist/Verstand) lässt sich offensichtlich nicht einfach auf seine Einzelbausteine wie Moleküle, Proteine etc. zurückführen. Letztere verrichten ihre Arbeit in einem weitestgehend entkoppelten Zustand vom Gesamtsystem. Wenn einzelne Moleküle, Proteine etc. ausfallen, bemerkt dies das Gesamtsystem Mensch in der Regel nicht bzw. nicht sofort. Erst wenn viele Grundbausteine betroffen sind, können sich Wirkungen bemerkbar machen. Und dass diese Einzelbausteine das Wesen eines Menschen nicht aus sich heraus ausmachen, wird sehr deutlich, wenn wir an den Tod denken. Etwas ist abhanden gekommen, von dem wir heute noch nicht genau sagen können, was dieses ,Etwas' tatsächlich genau ist.

Untersuchung von Bedingung 2:

Hier führten die Autoren als Beispiel das makroskopisch relevante Quantentunneln bei SQUIDS $^{135}$ an (ebd. 205), bei dem mikroskopische Prozesse (das Tunneln von Elektronen) makroskopisch relevante Auswirkungen haben (Stromflüsse ohne Verlust). Auch hier finden wir vergleichbare Prozesse bei Menschen, denn das Befinden und Wohler-

\footnotetext{
${ }^{135}$ SQUID steht für superconducting quantum interference device, zu dt. supraleitende Quanteninterferenzeinheit. Mit solchen Geräten können sehr kleine Magnetfeldänderungen gemessen werden. B.D. Josephson lieferte hierzu die theoretischen Überlegungen.
} 
gehen des makroskopischen Systems Mensch hängt von seinen Einzelbausteinen ab und kann von diesen wesentlich beeinflusst werden. Der Transformationsprozess von Energie (Abb. 35) respektive Information (Abb. 36) im Menschen lässt sich entsprechend darstellen.

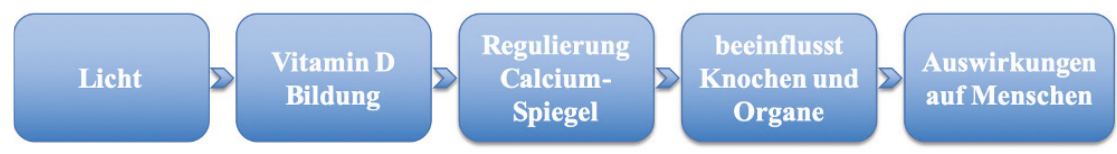

Abb. 35 | Beispiel: Einfluss einer mikroskopischen Energie auf das Makrosystem Mensch

Photonen tunneln in die Zellen und Nerven und lösen bio-chemische Prozesse aus. Sonnenlicht unterstützt den Körper zur Bildung von Vitamin D. Vitamin D ist an der Regulierung des Calciumspiegels beteiligt, der wiederum für den Aufbau von Knochen und Organen mitverantwortlich ist. Ein Mangel hat schließlich Konsequenzen für die Gesundheit des Menschen (Dusso u. a. 2005).

Informationen gekoppelt an Träger (Photonen, Moleküle etc.) treffen auf einen Menschen und fließen oder tunneln in das neue System, um dort neue Impulse auszulösen. Impulse, die über elektro-magnetische auf bio-chemische Prozesse einwirken und so Resonanz im Körper oder Gehirn bewirken; Resonanz, die durch den Menschen wahrgenommen werden kann und dessen Bewusstsein diese Wahrnehmungen schließlich interpretiert. Diese Prozesse werden in den Kapiteln Biologie und Neurowissenschaften eingehend behandelt. An dieser Stelle sei nur auf das Erkennen feindlicher Flieger und die bisher vorgestellten neurowissenschaftlichen Forschungen verwiesen.

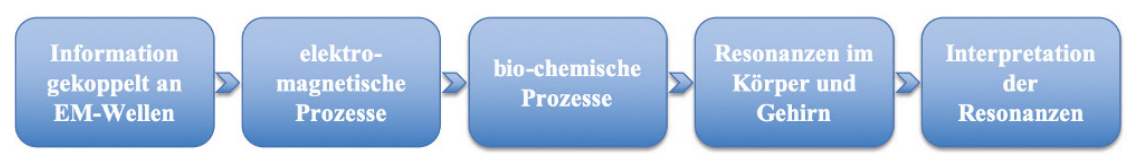

Abb. 36 | Transformation von Information

Als verallgemeinertes Modell wird hier gezeigt, wie Information sich über verschiedene Stufen von der Quantenebene bis zur Makroebene eines Menschen entfalten kann. (eigene Darstellung)

Untersuchung von Bedingung 3:

In diesem Punkt gehen die Autoren noch davon aus, dass thermische Bewegungen Quanteneffekte ,auswaschen' (ebd. 204). Diese noch sehr weitverbreitete Überzeugung geht davon aus, dass hohe Temperaturen (bereits wenige Kelvin über dem absoluten Nullpunkt) und die damit verbundene thermische Bewegung die Wechselwirkungen zwischen den Quantensystemen so fördert, dass die spezifischen Eigen-Zustände verloren gehen.

Punkt 3 scheint deshalb auf den ersten Blick Probleme aufzuwerfen. Wie jedoch noch zu zeigen sein wird, lassen sich quantenphysikalische Mechanismen bereits bei normalen Umgebungstemperaturen beobachtet. Beispielhaft sei schon einmal auf die QuantenTeleportation von Zeilinger's Gruppe (Ma u. a. 2012a) und auf den Nachweis von Tunnelprozessen in Bakterien von der Gruppe um Plenio (Chin u. a. 2013) verwiesen. Es 
kann sich deshalb hier um keine absolute Forderung nach tatsächlichen Tieftemperaturen handeln. Worauf es scheinbar letztlich ankommt, ist die Forderung nach Energieniveaus ${ }^{136}$ oder Erregungszuständen, die jeweils wechselwirken können und sich gegenüber dem Umfeld als widerstandsfähig erweisen. Daraus abgeleitet, drängt sich eine Schlussfolgerung auf (Greenstein und Zajonc 2005: 203-205). Die Forderung ...

\section{„The temperature must be low“" kann als relative Größe verstanden werden!}

denn jedes System und das mit ihm interagierende Umfeld hat jeweils spezifische Zustände, bei dem Verschränkung und Kohärenz möglich sind, deshalb ...

\section{Temperatur und Erregungszustand sind ,relativ' zu sehen. Relativ zu den betrachteten Systemen, die interagieren!}

Sichergestellt werden muss nur eine für die beteiligten Systeme stimmige Ebene, auf der Kohärenz möglich ist.

Greenstein und Zajonc formulieren damit drei Bedingungen, die relativ problemlos auf den Menschen und eigentlich alle lebenden Systeme übertragen werden können. Mit der Überwindung des Tieftemperatur-Paradigmas, das auch von Greenstein und Zajonc noch nicht gesehen wurde, sind Voraussetzung definiert, die jetzt Quantenverhalten in Makrosystemen denkbar erscheinen lassen.

\subsubsection{Dreizehn Bedingungen nach Walter von Lucadou}

Dass SyA die geeignete Methode darstellt, um mögliche nicht-lokale Effekte und damit die Hypothese einer Quantentheorie als Basismechanismus zu untersuchen (im Sinn der GQT als auch im Sinn der Physik), geht aus 13 von Walter von Lucadou aufgestellten Forderungen zur Untersuchung von Psi-Phänomenen hervor (Lucadou 2000). Denn im Grunde geht es bei SyA um heute noch nicht erklärbare Psi-Phänomene, nur dass sie signifikanter und wiederholbarer sind, als die bisher in dieser Kategorie untersuchten. Zudem entsprechen die Forderungen seiner Auflistung dem natürlichen Aufbau und Ablauf von SyA.

In Klammern ist meine Einschätzung ergänzt, inwieweit Aufstellungsarbeit der jeweiligen Forderung entspricht; mit einer Skala von 0 bis 10, wobei 0 der Forderung, gar nicht' entspricht und 10 die Forderung zu 100 \%' erfüllt.

${ }^{136}$ Ein Energieniveau in quantenphysikalischen Systemen entspricht bestimmten Energiewerten. Da immer mehrere solcher Energieniveaus für ein System existieren, lässt sich für jedes System ein Gesamtenergiespektrum definieren. Das niedrigste Energieniveau entspricht dem Grundzustand des Systems, alle anderen sind demzufolge angeregte oder sogenannte Erregungszustände. Etwas Vergleichbares gibt es in der Psychologie. Ein Mensch im Grundzustand ist i. d. R. ruhig, im Erregungszustand dagegen aktiv bis aggressiv. Eine konstruktive Wechselwirkung erfordert zunächst die Anpassung an den Erregungszustand des Gegenübers, wie es im NLP als Pacing gelehrt wird. 
Vorneweg formuliert Lucadou noch eine Metaforderung, auf die im Kapitel Verschränkung näher eingegangen wird (ebd. 15-16):

$$
\text { „Behandelt Psi nicht wie ein Signal!“ }
$$

Nun die 13 Forderungen:

1. „Sympathie, empathische Versuchsatmosphäre;

2. Epidemiologische Studien, Feldstudien;

3. Keine Akkumulation, kurze Runs;

4. Nahe am physikalischen Prozess, Fluktuationen, viele Kanäle;

5. Korrelationen zwischen physiologischen und psychologischen Variablen; (10)

6. Komplette Aufzeichnung des Prozesses - keine Datenreduktion;

7. Keine unabhängigen Ereignisse, besser Markoff-Ketten*;

8. Einfache Displays, eindeutige Instruktionen, keine Verschleierungen;

9. Dreifachblindheit**;

10. Organisierte Geschlossenheit des Experiments, räumlich und zeitlich;

11. Auswertung mit ,Distanz' (Daten reifen lassen);

12. Konzeptuelle Replikationen (identische Replikationen sind nicht möglich); (10)

13. Abhängige Variablen. “

* Von Lucadou, weist hier auf Ergebnisse aus PK-Experimenten (Präkognition) hin, bei denen reine Zufallsfolgen nicht so gut nachweisbar sind. Als Ursache vermutete er die Unabhängigkeit der Ereignisse, wobei Systemfluktuationen bei natürlichen Systemen immer interne Korrelationen aufweisen. Diesen Umstand setzt er in Beziehung mit Markoff-Ketten, die vergleichbar mit natürlichen Systemen, immer eine Geschichte haben. Es liegt nahe, die deutlich schwächeren Signifikanzen bei den bisher vorgestellten experimentellen Beispielen gegenüber von SyA, mit diesem Zusammenhang zu erklären. ** ,,Sowohl der Experimentator als auch der Teilnehmer sollte blind in Bezug auf die experimentellen Hypothesen sein (Doppelblind-Bedingungen) " (Lucadou 2000: 17). Diese reicht aber noch nicht aus: ,, Weil die operationale Kenntnis von früheren Experimenten einen realen Einfluss auf jedes weitere Experiment hat, müssen der Experimentator und der Versuchsteilnehmer auch blind in Bezug auf das operationale Resultat früherer Versuche sein " (ebd.). Auch hier lässt sich ein Zusammenhang mit unseren vorgestellten SyA- und Intuitions-Experimenten konstruieren. Viele von diesen Experimenten wurden mit (nach unserer Sprache) doppelter Verblindung durchgeführt. Im Sinne von Lucadou entsprach dies allerdings einer, Dreifachblindheit'.

\subsubsection{Bedingungen aus der GQT für mentale Verschränkungszustände}

Tressoldi und seine Gruppe, auf deren Experimente über nicht-lokale, soziale Interaktionen bereits hingewiesen wurde, fassten die Übertragung der physikalischen Gegebenheiten auf mentale Interaktionen im Sinne der GQT wie folgt zusammen (Giroldini u. a. 2016): 
,This theory predicts mind-to-mind and mind-to-matter non-local correlations similar to the entanglement phenomena observed in quantum physics if the following conditions are fulfilled:

1) A system is given, inside which subsystems can be identified. Entanglement phenomena will be best visible if the subsystems are sufficiently separated such that local observables pertaining to different subsystems are compatible.

2) There is a global observable of the total system, which is complementary to local observables of the subsystems.

3) The total system is in an entangled state. For instance, eigenstates of the global observable are typically entangled states.

The theory of Generalized Entanglement assumes that a distant social interaction between two persons who know each other must satisfy these requirements:

a) the two persons represent two subsystems of a single larger one created by their relationship, and

b) this relationship constitutes an entangled state, and furthermore that

c) the measurable psychological and physiological variables represent the system's comprehensive characteristic even though measured individually. "

Im Umgang mit mentalen Beobachtungsmerkmalen sehen sie allerdings noch Identifikations- und Operationalisierungsprobleme. So suchen sie aktuell nach Möglichkeiten Experimente zu kreieren, mit deren Hilfe die Bellsche Ungleichung (Wiseman 2014b, 2014a) überprüft werden kann; also der Unterschied zwischen klassischer Signalübertragung und quantenphysikalischer Korrelation. Mit der GHZ-Versuchsanordnung (Greenberger u. a. 2008, 1990) bedarf es nur noch der Sicherstellung einer physikalischen Verschränkung, womit ihre Suche nach Bell-Konfigurationen mittlerweile obsolet geworden ist, wie noch gezeigt werden wird.

\subsection{Anwendung der geforderten Bedingungen auf SyA}

Entsprechend der ersten heuristischen Betrachtung unter Kap. 7.1, wird zunächst eine zweite heuristische Annäherung unternommen, die sich auf die eben vorgestellten Bedingungen und Forderungen bezieht. Wir werden den aktuell akzeptierten Interpretationsrahmen der Quantenphysik probehalber verlassen und erste Vertiefungen bzw. Übertragungen vornehmen, um einen möglichen Argumentationsweg aufzuzeigen, wie die Phänomene in SyA entstehen könnten. Zum Zweiten werden wir noch einen Schritt weitergehen und den Betrachtungshorizont bis hin zu Organisationen erweitern.

Den heuristischen Bezug starten wir in umgekehrter Reihenfolge:

Die GQT (Atmanspacher u. a. 2002) und die von Giroldini u. a. (Giroldini u. a. 2016) vorgenommene Übersetzung für mentale, transpersonale Zustände lässt sich perfekt auf SyA übertragen. Ihre Fragen zur Bellschen Ungleichung und der Identifikations- und Operationalisierungsgestaltung werden sogar durch SyA beantwortet, sprich: SyA stellt die geeignete Untersuchungsmethode dar. Die technischen Problem-Aufstellungen sind 
hierfür in besonderer Weise geeignet. Liefern sie einerseits die Möglichkeit einer nachgelagerten eindeutigen Identifizierung, andererseits einfache, klar definierbare Messanordnungen, die nach Schlötter so gut wie von jeder Person eingenommen werden können (Schlötter und Simon 2005: 13).

Lucadou's Erkenntnisse (Lucadou 2000) korrelieren zu 100 \% mit Ergebnissen von SyA. Mit Nachdruck wird noch einmal auf die Deckungsgleichheit zwischen den 13 Forderungen und dem Ablauf bei SyA verwiesen. Damit lässt sich zunächst ebenfalls eine Entsprechung zur GQT herstellen und dem auf soziale Kontexte übertragenen quantenphysikalischen Formalismus (Atmanspacher u. a. 2002).

Greenstein und Zajonc formulierten drei Bedingungen für makroskopisches Quantenverhalten (Greenstein und Zajonc 2005: 203-205):

Beginnen wir mit Bedingung 3 und überlegen uns, wie diese Forderung in Verbindung mit SyA stehen könnte. Es ging hier um die Forderung nach niedriger Temperatur, die gleichzeitig als eine relative Größe in Bezug auf die beteiligten Systeme verstanden wird. Man könnte also sagen:

Die Repräsentanten in der SyA schwingen im gleichen Erregungszustand (ruhige gesammelte Aufmerksamkeit) und können so eine gemeinsame Kohärenz herstellen. Dies entspricht den Forderungen, die heute an die Präparation von Qubits gestellt werden: Die Anregung muss zur physikalischen Natur des Qubits passen; jetzt eben angewandt auf das bio-physikalische System Mensch.

Für Bedingung 2, bei der es um die Abhängigkeit der makroskopischen Größe von der mikroskopischen Energie ging, ließe sich folgender Zusammenhang herstellen: Aus den Untersuchungen und den Überlegungen zu Bakterien (Chin u. a. 2013), dass sich die Photonen in bewährter quantenphysikalischer Weise den für ihre Aufgabe und ihr Ziel effektivsten und schnellsten Weg durch das Bakterium wählen, könnte das Gleiche für Photonen bzw. Informationen bei menschlichen Systemen geschlussfolgert werden. Wir betrachten den Organismus damit als offenes Quantensystem, was im Gegensatz zum Verständnis der klassischen Biologie und Physik und auch zu einem Teil der Quantenphysiker steht.

Extrapolieren wir an dieser Stelle den Prozess noch bis zum System ,Organisation', so lässt sich etwa folgender Zusammenhang darstellen, der für ein erweitertes Verständnis zu Entscheidungstheorie und dem Überleben von Organisationen eminent wichtig ist (Abb. 37). Ideen, Ziele und Wünsche, auf mikroskopischer Ebene gekoppelt an Neuronen, treten in Kohärenz mit korrelierten Zuständen anderer Systeme (Menschen), erzeugen mentale Bilder oder Körpersymptome, beeinflussen dadurch das Verhalten ihrer Träger, welches wiederum auf das Verhalten von Gruppen und schließlich auf die gesamte Organisation wirkt. 


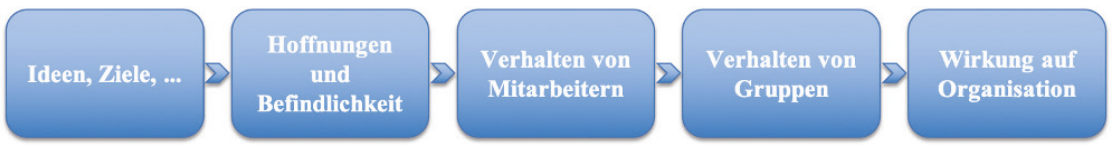

Abb. 37 | Beispiel für Einflüsse von Ideen auf das Makrosystem Organisation

Mentale Ideen und Ziele lösen Hoffnungen und Befindlichkeiten aus, welche das Verhalten von Mitarbeitern einer Organisation beeinflussen. Einzelne Mitarbeiter wiederum können das Verhalten von Gruppen und darüber schließlich eine Wirkung auf die Organisation als Ganzes entfalten. (eigene Darstellung)

Und last but not least extrapolieren wir auch Bedingung 1, bei der es um eine ausreichende Entkopplung des Makrosystems von seinen Einzelbausteinen geht, über den Menschen hinaus bis hin zu Organisationen, so lässt sich festhalten: Die Organisation ist nicht gleich der einzelne Mitarbeiter, eine Idee oder ein Ziel. Gibt es keine Ideen, keine Ziele oder fällt ein Mitarbeiter aus, kann deren Ausfall über mehr oder weniger lange Zeiträume kompensiert werden. Erst bei ausreichender Ausdehnung dysfunktionaler (dekohärenter) Zustände fängt das Gesamtsystem an Schaden zu nehmen.

Wie wir sehen, lassen sich die Bedingungen recht einfach über das Makrosystem Mensch hinaus bis hin zu von ihm geschaffenen Organisationen oder gar Kulturen extrapolieren. Sicher und ohne Widerspruch auszulösen, lassen sich diese Zusammenhänge einfach als Analogie verstehen. Wir werden aber im Weiteren sehen, dass es sich mitnichten um eine reine Analogie handeln muss.

In Summe drängt sich in jedem Fall die Frage in den Vordergrund: Reichen die eben aufgeführten Erklärungen, um quantenphysikalische Effekte als Grundlage für SyA plausibel erscheinen zu lassen? Die Antwort von Experten wird zu diesem Zeitpunkt wohl recht eindeutig ausfallen. NEIN! Die eben vorgenommenen Übertragungen erscheinen aus konventioneller Sicht zu beliebig und viel zu weit hergeholt.

Soll der Weg des Erklärungsansatzes tatsächlich erfolgreich zu Ende gebracht werden, ergibt sich zwingend die Notwendigkeit folgende weiterführende Fragen präzise beantworten zu können:

- Sind Quanteneffekte (Verschränkungen, Teleportation etc.) bei Umgebungstemperatur und in Makrosystemen möglich?

- Lassen sich Quantenprozesse in biologischen Systemen beobachten und beschreiben?

- Lassen sich bei Menschen (Körper und Gehirn) quantenmechanische Wechselwirkungen definieren und diagnostizieren?

- Insbesondere, gibt es eine Möglichkeit, wie Menschen das Problem der Wahrnehmung verschränkter Zustände lösen können?

- Lässt sich schließlich eine lückenlose Prozesskette von der Mikro- bis zur Makrowelt beschreiben, in der Informationen transportiert bzw. zugänglich gemacht werden können? 
Mit erfolgreicher Beantwortung dieser Fragen wäre Hauptkategorie 4 und in Folge Hypothese 4 (Kap. 2.1) und somit der letzte Baustein zur (natur-)wissenschaftlichen Legitimation gelegt.

Genau dieser Versuch soll im weiteren Verlauf unternommen werden und mit ...

$$
\begin{gathered}
\text { „Alles, aber auch Alles ist Quantenphysik, } \\
\text { und Alles ist mit Allem verbunden“" } \\
\text { (John Archibald Wheeler) }
\end{gathered}
$$

überprüfen wir, ob sich diese These von Wheeler durch SyA bestätigen lässt.

Open Access Dieses Kapitel wird unter der Creative Commons Namensnennung 4.0 International Lizenz (http://creativecommons.org/licenses/by/4.0/deed.de) veröffentlicht, welche die Nutzung, Vervielfältigung, Bearbeitung, Verbreitung und Wiedergabe in jeglichem Medium und Format erlaubt, sofern Sie den/die ursprünglichen Autor(en) und die Quelle ordnungsgemäß nennen, einen Link zur Creative Commons Lizenz beifügen und angeben, ob Änderungen vorgenommen wurden.

Die in diesem Kapitel enthaltenen Bilder und sonstiges Drittmaterial unterliegen ebenfalls der genannten Creative Commons Lizenz, sofern sich aus der Abbildungslegende nichts anderes ergibt. Sofern das betreffende Material nicht unter der genannten Creative Commons Lizenz steht und die betreffende Handlung nicht nach gesetzlichen Vorschriften erlaubt ist, ist für die oben aufgeführten Weiterverwendungen des Materials die Einwilligung des jeweiligen Rechteinhabers einzuholen.

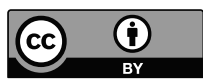

